\title{
Incidentaloma: Pagetic Scapula
}

\author{
Fahad Al Lhedan* and Anas Hamdoun \\ Department of Medical Imaging, Saudi Arabia
}

*Corresponding author: Fahad Al Lhedan, Medical Imaging Department, King Abdullah bin Abdulaziz University Hospital, Riyadh, Saudi Arabia.

Received Date: April 17, 2019

Published Date: April 26, 2019

\begin{abstract}
Osseous Paget's disease is the second most common remodeling bone disease in elderly patients after osteoporosis [1]. The prevalence of bone Paget's disease increases with age and there is male's predominance. Although Paget's disease of the bone is usually silent, however, various symptoms and serious complications could occur secondary to it [2,3]. We are reporting a rare case of a monostotic Paget's disease that involves only the scapula. The scapular lesion was noted initially, incidentally, on the chest plain film with radiographic features suggestive of Paget's disease. Thereafter, additional studies for further evaluation were done by preforming dedicated radiographs of the right scapula and whole-body bone scintigraphy with imaging findings compatible with this diagnosis possibility. Alkaline phosphatase was significantly high and serum calcium and phosphorus were within the normal range supporting the presence of Paget's disease.
\end{abstract}

\section{Introduction}

Paget's disease of the bone is of uncertain etiology that might involve only a solitary bone, monostotic, or more than one bone can be affected, polyostotic [4,5]. It is primarily an osteoclast disorder characterized by focal alteration in bone remodeling with resultant abnormal osseous structures formation. Patients with Paget's disease are usually symptoms-free, and they are discovered by chance, but this is not always the case. Multiple skeletal and extra skeletal manifestations and complications be a result of this disorder. Diagnosis of Paget's disease is usually made based on the radiographic and scintigraphic imaging findings in addition to the laboratory investigations. Medical therapy is the main stay for treatment and surgery is occasionally needed if there are any complications.

\section{Case presentation}

This is a 70-year-old elderly diabetic and hypertensive male patient who was asymptomatic, and he has had a chest radiograph as a part of routine follow-up. An incidental note was made of right scapular abnormality, which was further evaluated by dedicated radiographs of the right scapula. The radiographs displayed a relatively diffuse sclerosis, bone expansion and trabecular and cortical thickening. There was no bone destruction, cortical breakthrough, aggressive appearing periosteal reaction or an associated soft tissue component. Delayed phase whole body bone scan was done for disease extent assessment showing diffuse intense right scapular radiotracer uptake. No other foci of abnormal increased radiotracer uptake were identified throughout the axial or appendicular skeleton. Alkaline phosphatase was more than two times higher than normal measuring 238U/L and serum calcium and phosphorus were normal measuring $2.23 \mathrm{mmol} / \mathrm{L}$ and $0.94 \mathrm{mmol} / \mathrm{L}$, respectively. Diagnosis of monostatic scapular Paget's disease was made based on the constellation of the imaging findings and laboratory investigations Figures (1-3).

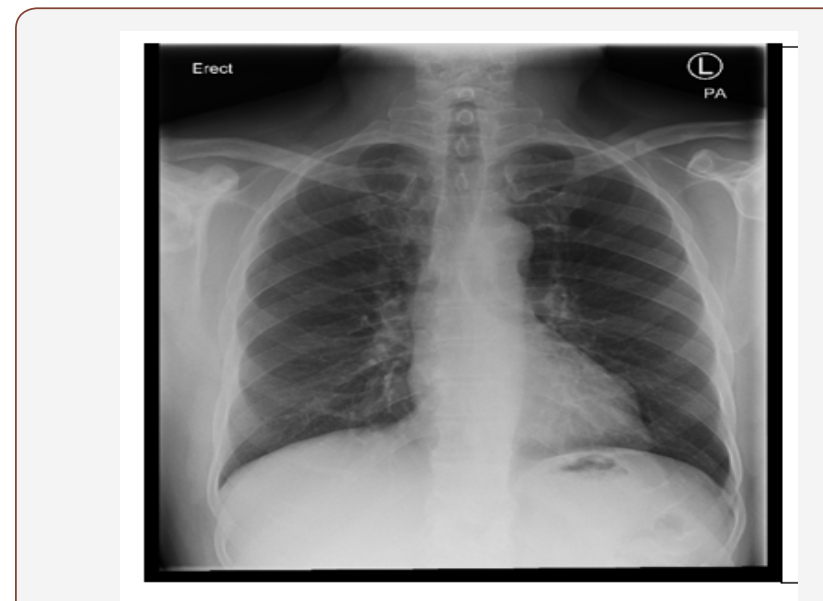

Figure 1: Frontal chest radiograph: diffuse sclerosis, trabecular and cortical thickening of the partially visualized right scapula. 


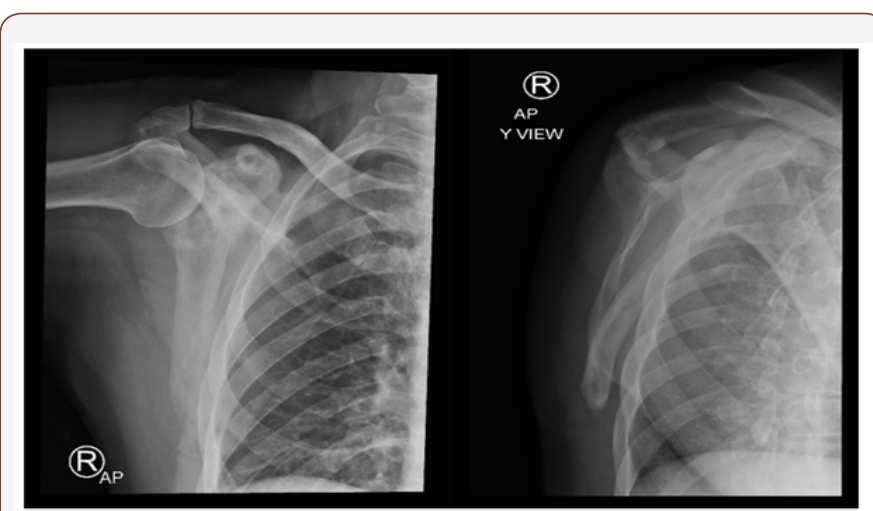

Figure 2: Radiographs of the right scapula: diffuse sclerosis, bone expansion and trabecular and cortical thickening with no bone destruction, cortical breakthrough, aggressive periosteal reaction or soft tissue component.

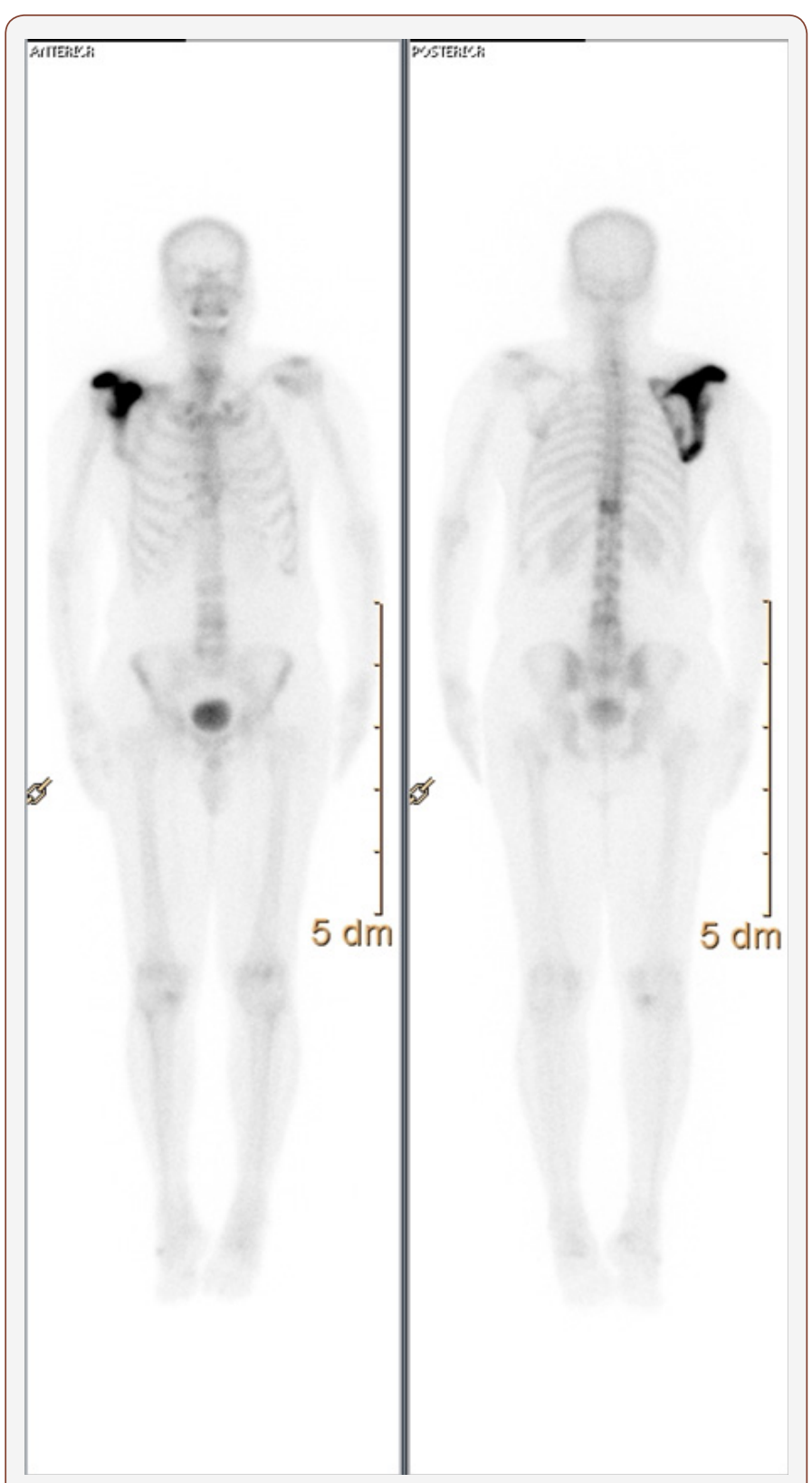

Figure 3: Whole body delayed phase bone scan: diffuse intense right scapular radiotracer uptake with no other foci of abnormal increased radiotracer uptake in the axial or appendicular skeleton.

\section{Discussion}

Paget's disease has been misnomerly called osteitis deformans due to the believe that it results from a chronic inflammation but it is actually a chronic, non-inflammatory and localized boneremodeling disorder that affects widespread and non-contiguous areas of the skeleton therefore using the term osteodystrophia deformans is more appropriate [6,7]. Paget's disease is unusually encountered before the fourth decade of life and it is more in males with a suggestion that multiple genetic and environmental factors play a role in the development of this disorder [2,8]. Osteolysis predominates in the early course of the disease causing focal lytic lesions. Osteosclerosis develops subsequently leading to the formation of mixed lytic and sclerotic lesions, bone expansion, trabecular and cortical thickening [2,6]. Plain radiograph is the initial diagnostic modality tool. Whole body bone scintigraphy is highly sensitive by revealing the pattern of intense radiotracer uptake, but it is not specific, and its usefulness is evident in determination the exact disease extension [8].

The serum calcium and phosphorus are usually within normal limits and the serum total and bone specific turnover marker, namely the alkaline phosphatase, is usually significantly elevated in pagetic patients. Alkaline phosphatase value correlates well with the degree of bony involvement and its measurement is informative in disease activity assessment, response to treatment and in exclusion the biochemical relapse [8]. Alkaline phosphatase is an inexpensive and readily available parameter, and therefore it is widely used for Paget's disease activity monitoring. However, it is unreliable in the presence of a hepatobiliary disease [8]. Pelvic bones, lumbar vertebrae, femora, tibiae as well as skull are the most commonly involved sites while the upper extremities, clavicles, ribs and scapulae are less frequently affected [2]. The most common musculoskeletal manifestations of Paget's disease include osteoarthritis, fractures and bowing deformities in a descending order of frequency. Sarcomatous transformation is fortunately very rare but it is a possible complication $[2,8]$. The neurologic symptoms such as hearing loss and headache are uncommon. High output heart failure is an infrequently occurrence cardiovascular sequela. Hypercalcemia and hyperuricemia are seldom metabolic derangement that can be also identified $[2,8]$. Medical therapy with potent amino bisphosphonates is very effective in treating Paget's disease and the surgical choice for fracture stabilization, corrective osteotomy and total joint replacement is required whenever there is clinical indication [2].

\section{Conclusion}

Monostotic Paget's disease of the scapula is an extremely rare. Diagnosis of Paget's disease is based on the biochemical markers and the pathognomonic radiographic features along with the scintigraphic findings. Paget's disease is a medically treatable illness and surgery might be indicated in some complicated circumstances. Long-term close follow up is mandatory to ensure complete resolution and to rule out biochemical disease recurrence or possible malignant transformation. 


\section{Acknowledgement}

None.

\section{Conflicts of interest}

The authors declare that there are no conflicts of interest regarding the publication of this paper.

\section{References}

1. Suwitda Cholitkul, Suteevan Cholitkul, Kamal Masaki, Dalia Elsemary (2008) Paget's Disease of Bone: A Case Report and Review of the Literature. Journal of The American Medical Directors Association 9(3): B20.

2. Joseph LShaker (2009) Paget's disease of Bone:A Review of Epidemiology, Pathophysiology and Management. Ther Adv Musculoskelet Dis 1(2): 107-125.
3. AVS Anil Kumar, PG Kumar, MS Prakash, V Tewari, H Sahni, et al. (2013) Paget's disease diagnosed on bone scintigraphy: Case report and literature review. Indian J Nucl Med 28(2): 121-123.

4. Esin Eray, Ramazan Sarı, Mustafa Kemal Balcı (2004) Isolated Paget's Disease of Frontal Bone: A Case Report. 3: 121-123.

5. Wegierska M, Ignaczak P, Zalewska J, Jeka S (2011) Paget's disease: case report. Annals Academiae Medicae Stetinensis 57(3): 64-69.

6. Zinon T Kokkalis, Stefania Kokkineli, Ilias D Iliopoulos, Panayiotis D Megaloikonomos, Andreas F Mavrogenis, et al. (2018) Monostotic Paget's disease of the radius: A case report. Clinical Cases in Mineral and Bone Metabolism 15(2): 267-270.

7. Y Uday Shankar, Satya Ranjan Misra, Daniel Alex Vineet, Pavitra Baskaran (2013) Paget disease of bone: A classic case report. Contemp Clin Dent 4(2): 227-230.

8. Stephen Paul Tuck, Robert Layfield, Julie Walker, Babitha Mekkayil, Roger Francis (2017) Adult Paget's disease of bone: a review. Rheumatology (Oxford) 56(12): 2050-2059. 